\title{
Analytical Study on the Safety of High Speed Railway Vehicle on Excited Tracks*
}

\author{
Kazuhiko NISHIMURA **, Yoshiaki TERUMICHI ***, \\ Tsutomu MORIMURA ** and Kiyoshi SOGABE *** \\ ** Technology Research and Development Department (Komaki), Central Japan Railway Company \\ 1545-33 Ohyama, Komaki-shi, Aichi, Japan \\ E-mail:kazuhiko.nishimura@jr-central.co.jp \\ *** Department of Mechanical Engineering, Sophia University \\ 7-1 Kioi-cho Chiyodaku Tokyo, Japan
}

\begin{abstract}
A railway is organized by a variety of individual technologies, and functions safely and properly as a system, therefore it is necessary for the system safety to study each potential case of disasters caused by earthquakes. Recent reports indicate that railway vehicles could be derailed solely by the ground motions of earthquakes with no fatal damages of vehicles or tracks. Based on the reports and facts, we believe that we should further study the derailment mechanism of a high speed railway vehicle excited by large seismic motions, to pursue to minimize the risk of railway system against large earthquakes. At the start of the study, we developed our original vehicle dynamics simulation and then employed it for numerical analyses. At the present stage, through the analyses, we obtained the following major outcomes. (1) Most of derailments are brought as the result of the rocking motion of a vehicle by track excitations underneath. Interestingly, the derailing motions are observed similarly regardless of vehicle speed. (2) By contrast, the excitation amplitudes for derailment are influenced by vehicle speed particularly in lower input frequencies. This can be explained by the sensitivity of the relative wheel/rail slide due to creepage. (3) The excitation amplitudes for $30 \mathrm{~mm}$ of wheel lift are relatively independent of vehicle speed. (4) The wheel/rail slide strongly depends on the friction coefficient if a vehicle stationed, being relatively independent of the friction coefficient at higher speeds.
\end{abstract}

Key words: Railway, Earthquake, Seismic Motion, Derailment, Rocking, Multibody Dynamics, Simulation

\section{Introduction}

Due to the geographic reason of being located right above earthquake nests, Japan has had a considerable number of earthquakes. Consequently, numerous earthquake disasters have been experienced. Through tragic experiences, the necessity of the study and research on earthquake disaster prevention has been a high-profile issue and many efforts have been made to minimize possible disasters. In the railway industry, railway infrastructures have been continually reinforced and a new alert system has been employed to regulate the operating system so as to stop trains immediately after a large earthquake occurs. It should be noted that a railway is organized by a variety of individual technologies and functions safely and properly as a system. Therefore, every potential disaster should be carefully studied and examined from different points of view. Recent reports imply that rail vehicles

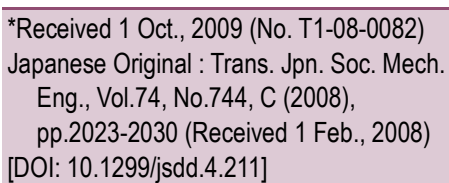

Copyright $(\mathcal{2} 2010$ by JSME 
could derail solely by the excitation of earthquakes. Nagase ${ }^{[1]}$ reported that a number of trains were derailed, including the ones in operation, directly due to the track excitations in the Kobe Earthquake in January 1995. At Mid Niigata Prefecture Earthquake in October 2004 , a Shinkansen train was derailed while being operated at the speed of $200 \mathrm{~km} / \mathrm{h}$, which was the first case of the derailment of a Japanese high speed train under commercial operation through the long history of these trains. As reported in Ref [2], the horizontal ground motion caused by the earthquake was concluded to be the major cause of the derailment. Based on the reports and facts, we believe that we should further study the derailment mechanism of high speed railway vehicles excited by large seismic motions both theoretically and experimentally.

Historically, there are many studies that focus on the general safety and derailment issues of rail vehicles; Ishida and Matsuo ${ }^{[3]}$ summarized the safety criteria for the assessment of general derailments. Miyamoto ${ }^{[4]}$ reviewed the mechanisms of derailments for general cases. Matsudaira and Yokose ${ }^{[5,6]}$ studied a jumping or hunting derailment of high speed rail vehicles and suggested criteria for this type of derailment, which may be caused by severe impacts of troubled wheel-set to rails. Matsuo ${ }^{[7]}$ and Ishida ${ }^{[8]}$ studied the safety of a high speed rail vehicles involving high frequency fluctuation of wheel load caused by short-wavelength rail irregularities and demonstrated the relationship between the length of time that the derailment quotient exceeded the criterion and the distance of the wheel-lift above the rail.

On the other hand, there are few studies for the specific vehicle safety in the case of earthquakes. At the time of building the bridge connecting Honshu-Shikoku, the vehicle safety on excited tracks was rigorously studied ${ }^{[9]}$. By employing a unique simplified 3 DoF vehicle model of Shinkansen, safety limit amplitudes of excitations were suggested. Note that wheel lift and wheel/rail slide were not allowed in this simplified model. Kunieda ${ }^{[10]}$ analyzed turnover motions of rail vehicles on earthquakes by using a simplified two dimensional vehicle model, and presented the minimum external accelerations acting on vehicle bodies to cause the wheel loads to disappear. In recent studies, Miyamoto ${ }^{[11]}$ introduced a vehicle-track model for the safety assessment of a complete vehicle and pointed out those horizontal excitations have a much more severe impact on vehicle safety than vertical ones.

In this paper, we focus on the study for the vehicle safety in terms of the dynamic stability and the derailment mechanism directly caused by track excitations. We developed a 13 DoF planer vehicle dynamics model ${ }^{[12]}$ to represent the entire dynamic response of a rail vehicle and rails underneath. Then through the analytical approach, we suggest the fundamental derailment mechanism and also discuss the effect of vehicle speed on the derailment mechanism by specifically analyzing the two dimensional wheel/rail motions and forces.

\section{Nomenclature}

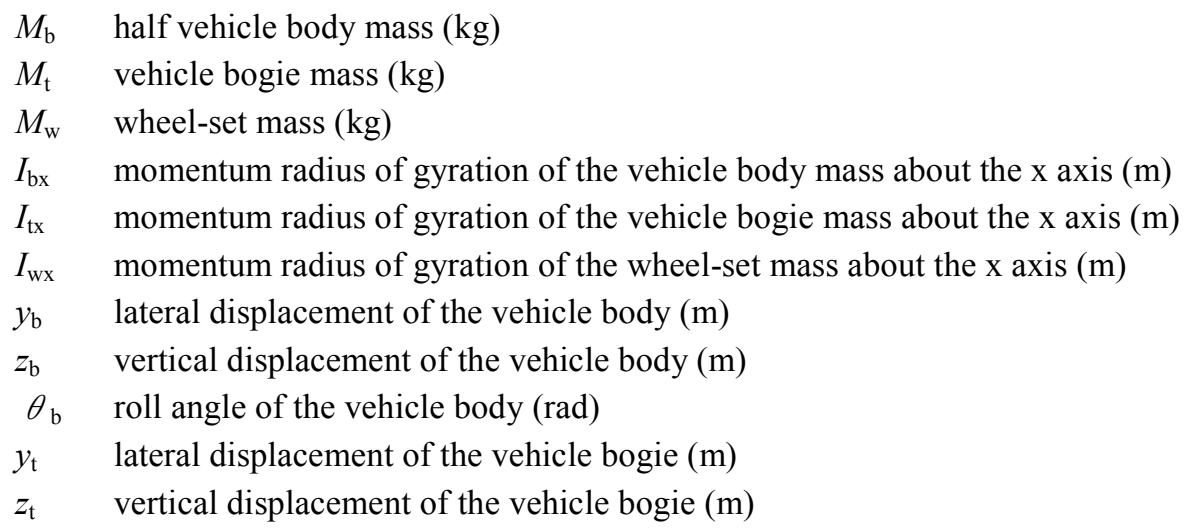


$\theta_{\mathrm{t}} \quad$ roll angle of the vehicle bogie ( $\left.\mathrm{rad}\right)$

$y_{\mathrm{wi}} \quad$ lateral displacement of the wheel-set (m) $i=1,2$

$z_{\mathrm{wi}} \quad$ vertical displacement of the wheel-set (m) $i=1,2$

$\theta_{\mathrm{wi}} \quad$ roll angle of the wheel-set (rad) $i=1,2$

$k_{1} \quad$ vertical primary suspension stiffness $(\mathrm{N} / \mathrm{m})$

$k_{2} \quad$ vertical air suspension stiffness $(\mathrm{N} / \mathrm{m})$

$k_{3} \quad$ lateral air suspension stiffness $(\mathrm{N} / \mathrm{m})$

$k_{\mathrm{wy}} \quad$ lateral primary suspension stiffness $(\mathrm{N} / \mathrm{m})$

$c_{1} \quad$ vertical primary suspension damping constant $(\mathrm{Ns} / \mathrm{m})$

$c_{2} \quad$ vertical air suspension damping constant $(\mathrm{Ns} / \mathrm{m})$

$c_{3} \quad$ lateral air suspension damping constant $(\mathrm{Ns} / \mathrm{m})$

$c_{\mathrm{wy}} \quad$ lateral primary suspension damping constant $(\mathrm{Ns} / \mathrm{m})$

$b_{1} \quad$ half of the distance between the left and right side of the primary suspensions $(\mathrm{m})$

$b_{2} \quad$ half of the distance between the left and right side of the air suspensions (m)

$b_{\mathrm{w}} \quad$ half of the distance between the left and right contact point of the wheel/rail (m)

$h_{\mathrm{b}} \quad$ vertical distance between the center of gravity of the vehicle body and the air suspension (m)

$h_{\mathrm{s}} \quad$ vertical distance between the center of gravity of the vehicle bogie and the air suspension (m)

$h_{\mathrm{t}} \quad$ vertical distance between the center of gravity of the vehicle bogie and the wheel-set (m)

$r_{\mathrm{w}} \quad$ wheel radius $(\mathrm{m})$

$F_{1 \mathrm{i}} \quad$ vertical force acting between the vehicle bogie and the wheel-set (N) $i=1,2$

$F_{2 \mathrm{i}} \quad$ vertical force acting between the vehicle body and the bogie $(\mathrm{N}) i=1,2$

$F_{3} \quad$ lateral force acting between the vehicle body and the bogie (N)

$F_{4} \quad$ lateral force acting between the vehicle bogie and the wheel-set (N)

$F_{5} \quad$ vertical force acting at the contact point of the traction link( vehicle body) and the bogie frame $(\mathrm{N})$

$F_{6 \mathrm{i}} \quad$ lateral slide force acting between the vehicle body and the bogie (N) $i=1,2$

$F_{7} \quad$ vertical slide force acting between the vehicle body and the bogie (N)

$P_{\mathrm{i}} \quad$ dynamic wheel load (N) $i=1,2$

$Q_{\mathrm{i}} \quad$ dynamic lateral force (N) $i=1,2$

$z_{1 \mathrm{i}} \quad$ vertical primary suspension travel $(\mathrm{m}) i=1,2$

$z_{2 \mathrm{i}} \quad$ vertical air suspension travel (m) $i=1,2$

$z_{2 \mathrm{~s}} \quad$ vertical clearance inside of the air suspension (m)

$\mu_{\mathrm{y}} \quad$ lateral friction coefficient for the slide force at the air suspension contact area

$y_{3} \quad$ lateral air suspension travel (m) $i=1,2$

$y_{3 \mathrm{~s}} \quad$ lateral clearance between the vehicle body and the bogie $(\mathrm{m})$

$\mu_{\mathrm{z}} \quad$ vertical friction coefficient for the slide force at the contact area of the bump stop

$y_{\mathrm{ri}} \quad$ lateral displacement of the rail (m) $i=1,2$

$z_{\mathrm{ri}} \quad$ vertical displacement of the rail (m) $i=1,2$

$k_{\mathrm{ry}} \quad$ lateral stiffness between the rail and the ground $(\mathrm{N} / \mathrm{m})$

$k_{\mathrm{rz}} \quad$ vertical stiffness between the rail and the ground $(\mathrm{N} / \mathrm{m})$

$c_{\mathrm{ry}}$ lateral damping constant between the rail and the ground $(\mathrm{Ns} / \mathrm{m})$

$c_{\mathrm{rz}} \quad$ vertical damping constant between the rail and the ground $(\mathrm{Ns} / \mathrm{m})$

$N_{\mathrm{i}} \quad$ normal force acting at the wheel/rail contact point (N) $i=1,2$

$T_{\mathrm{i}} \quad$ tangential force acting at the wheel/rail contact point (N) $i=1,2$ 
$P_{s t} \quad$ static wheel load (N)

$\alpha_{\mathrm{i}} \quad$ variable wheel/rail contact angle ( $\left.\mathrm{rad}\right) i=1,2$

$r_{\mathrm{i}} \quad$ variable wheel/rail contact radius $(\mathrm{m}) i=1,2$

$b b_{\mathrm{i}} \quad$ half of the variable distance between the left and right contact point of the wheel/rail (m) $i=1,2$

$y_{\mathrm{g}} \quad$ lateral displacement of the ground as the input excitation (m)

\section{Vehicle dynamics simulation}

\subsection{Vehicle model}

We propose a 13 DoF vehicle-track dynamics model composed of a 9 DoF half car model and a 4 DoF track model to analyze the entire dynamic response of a rail vehicle and rails. The vehicle and rails are excited by the horizontal sine displacement through the ground directly under the rails. The reasonably simplified dynamics model should be suitable for studying the derailment mechanism due to earthquake ground motions.

The half car model shown in Fig.1 includes suspension springs and dampers acting among the vehicle body mass $\left(M_{\mathrm{b}}\right)$, the bogie mass $\left(M_{\mathrm{t}}\right)$ and the two wheel-sets masses $\left(M_{\mathrm{w}}\right)$, and captures the vertical and lateral motions coupled to roll motions of the vehicle. The motions are described by the coordinates; $\left(y_{\mathrm{b}}, z_{\mathrm{b}}, \theta_{\mathrm{b}}\right)$ for the vehicle body, $\left(y_{\mathrm{t}}, z_{\mathrm{t}}, \theta_{\mathrm{t}}\right)$ for the bogie and $\left(y_{\mathrm{w} 1}, z_{\mathrm{w} 1}, \theta_{\mathrm{w} 1}, y_{\mathrm{w} 2}, z_{\mathrm{w} 2}, \theta_{\mathrm{w} 2}\right)$ for the two wheel-sets. Note that the motions of two wheel-sets are not independent, thus $\left(y_{\mathrm{w} 1}, z_{\mathrm{w} 1}\right.$ and $\left.\theta_{\mathrm{w} 1}\right)$ are equal to $\left(y_{\mathrm{w} 2}, z_{\mathrm{w} 2}\right.$ and $\left.\theta_{\mathrm{w} 2}\right)$ respectively. The diagram of the forces acting on the half car model is shown in Fig.2 and the dynamic equation is given by Eq. (1).

$$
\mathbf{M} \ddot{\tilde{e}}=\mathbf{F}
$$

Where, $\mathbf{M}, \mathbf{F}$, and $\widetilde{e}$ represent mass and inertia metrics, forces and moments acting on the vehicle, and the position and rotation vectors are given by Eq.(2), respectively.

$$
\widetilde{e}=\left(y_{b}, y_{t}, y_{w 1}, y_{w 2}, z_{b}, z_{t}, z_{w 1}, z_{w 2}, \theta_{b}, \theta_{t}, \theta_{w 1}, \theta_{w 2}\right)
$$

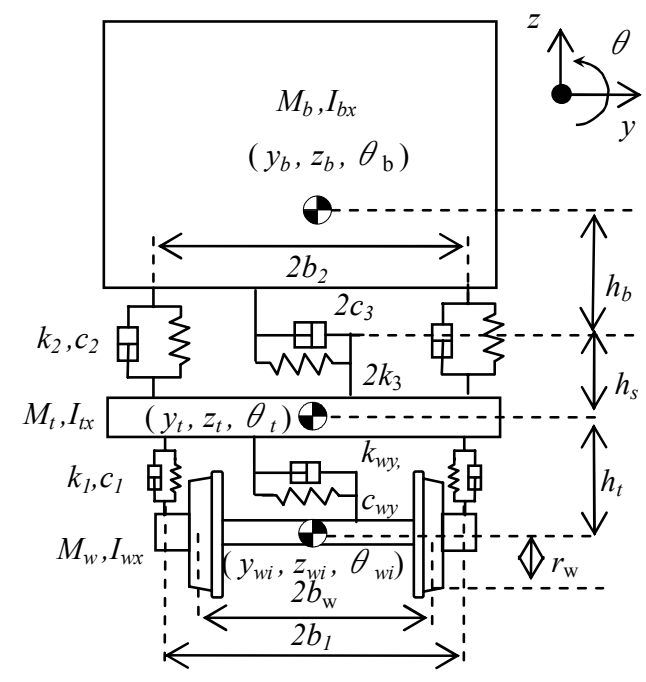

Fig.1 Half car model for 13 DoF vehicle-track dynamics model

Due to large vehicle motions, the forces considered in the dynamic equations exercise strong nonlinearities of spring stiffness, dampers and bump stops. The basic idea for the formulation of nonlinearities in the equations is mentioned as follows. Specification 
parameters are listed in Table 1.

(a) Regarding $F_{11}$ and $F_{12}$, the vertical stiffness of the primary suspension increases if the vertical displacement of the bogie relative to the wheel-set is larger than the clearance between those two because of their metal contact.

(b) Regarding $F_{21}$ and $F_{22}$, the vertical stiffness of the air suspension increases if the downward displacement of the vehicle body relative to the bogie is larger than the clearance of the inside of the air suspension.

(c) Regarding $F_{3}$, the lateral stiffness of the air suspension increases if the lateral displacement of the vehicle body relative to the bogie is larger than the clearance between those two because of the contact between the bump stop and the vehicle body.

(d) Regarding $F_{4}$, the lateral stiffness of the primary suspension increases if the lateral displacement of the bogie relative to the wheel-set becomes large because of the nonlinearity of the suspension rubber.

(e) As an extra force, $F_{5}$ is assumed if the upward displacement of the vehicle body relative to the bogie exceeds the clearance between those two because of the metal contact between the traction link (vehicle body) and the bogie frame.

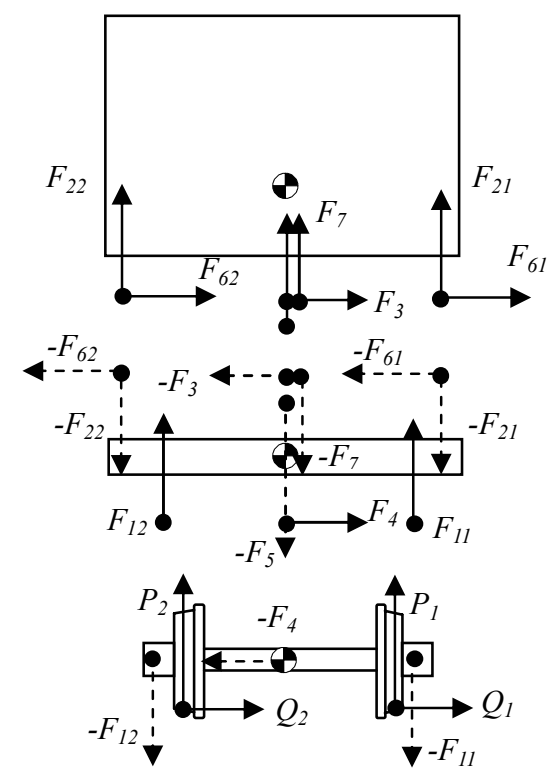

Fig.2 The diagram of forces acting on a vehicle

In this paper, besides what is mentioned, unique models are applied. Slide forces are assumed to be yielded by large vehicle motions at the contact areas between the vehicle body and the bogie, if there exists relative velocities between them.

(f) $F_{61}$ and $F_{62}$ act as described in Fig.3. On the condition that the air suspension is vertically compressed to the lower stroke limit and simultaneously the upper side of the air suspension (vehicle body) has a horizontal velocity relative to the lower side (bogie), the slide friction force defined by Eq. (3) or (4) is supposed to act toward the horizontal direction at the contact area.

$$
\begin{aligned}
& \text { if } z_{2 i} \leq-z_{2 s} \quad F_{6 i}=-\operatorname{sign}\left(\dot{y}_{b}-\dot{y}_{t}\right) \mu_{y}\left|F_{2 i}\right| \\
& \text { if } z_{2 i}>-z_{2 s} \quad F_{6 i}=0
\end{aligned}
$$

(g) $F_{7}$ acts as described in Fig.4. On the condition that the lateral displacement of the vehicle body relative to the bogie exceeds the clearance between those two and the vehicle body simultaneously has a vertical velocity to the bogie, the slide friction force defined by 
Eq. (5) or (6) is supposed to act toward the vertical direction at the contact area of those two.

$$
\begin{aligned}
& \text { if }\left|y_{3}\right| \geq y_{3 s} \quad F_{7}=-\operatorname{sign}\left(\dot{z}_{b}-\dot{z}_{t}\right) \mu_{z}\left|F_{3}\right| \\
& \text { if } \quad\left|y_{3}\right|<y_{3 s} \quad F_{7}=0
\end{aligned}
$$

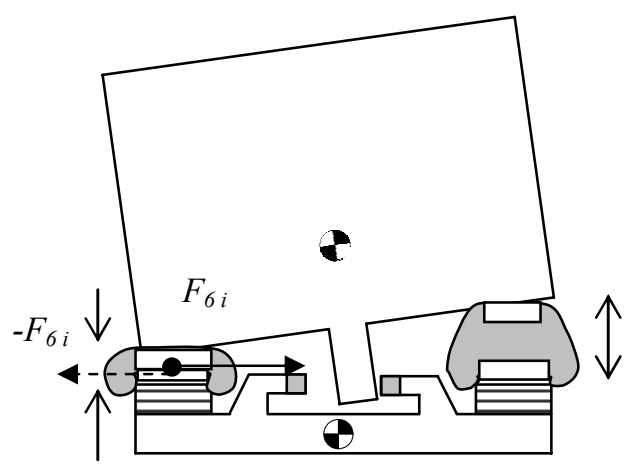

Fig.3 Slide force at air suspension

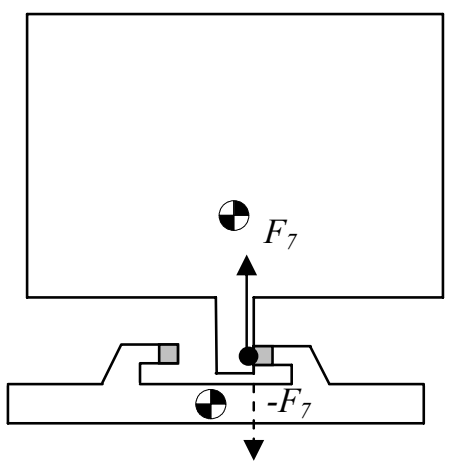

Fig.4 Slide force at bump stop

\subsection{Track model}

The track model shown in Fig.5 includes springs and dampers acting between the rails and the ground, and captures the vertical and lateral motions of the rails. The motions are described by the coordinates $\left(y_{r l}, z_{r 1}, y_{r 2}, z_{r 2}\right)$ for both rails. The ground position is described by the one coordinate $\left(y_{g}\right)$ as the input displacement. This model captures the rigid body dynamics in the roll plane and ignores roll, yaw and pitch motions. The masses of rails are ignored because they are small compared with the vehicle mass.

In the case of wheel/rail contact, the dynamic equations of rails are given by Eqs.(7) and (8) with use of the dynamic wheel load and the lateral force; otherwise, the dynamic wheel load and the lateral force are zero, and the rail stays at the same position. The wheel load and the lateral force are expressed by Eqs.(9) and (10) with use of the normal and tangential forces, respectively.

$$
\begin{aligned}
& c_{r y}\left(\dot{y}_{r i}-\dot{y}_{g}\right)+k_{r y}\left(y_{r i}-y_{g}\right)+Q_{i}=0 \\
& c_{r z} \dot{z}_{r i}+k_{r z} z_{r i}+\left(P_{i}-P_{s t}\right)=0 \\
& Q_{i}=N_{i} \sin \alpha_{i}+T_{i} \cos \alpha_{i} \\
& P_{i}=N_{i} \cos \alpha_{i}-T_{i} \sin \alpha_{i}
\end{aligned}
$$




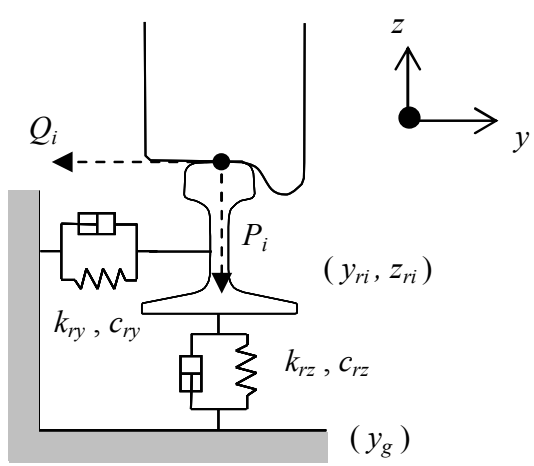

Fig.5 Track model for 13 DoF vehicle-track dynamics model

For simplicity, the wheel/rail contact is assumed to be at one point; otherwise, the wheel lifts off a rail. The transition from one point contact to wheel lift off is judged by the vertical wheel/rail geometry. If a wheel contacts a rail, those data of the contact angle of wheel/rail, the contact radius of the wheel, and the horizontal distance between the contact point and the center of the track are calculated by a prepared table based on the horizontal displacement of the wheel relative to the rail.

The wheel/rail tangential force is calculated by the creep force based on Kalker's theory and Lévi-Chartet Law if a vehicle has forward speed; otherwise i.e. a vehicle is still, the tangential force is calculated based on the coulomb friction law. The friction coefficient between wheel and rail is assumed to be 0.3 .

The wheel/rail normal force is calculated by the sum of wheel load and lateral force of the normal direction components. If a wheel lifts off its rail, the wheel/rail normal force becomes zero. As the procedure of determination of rail position, rail lateral position is determined by Eq. (7) and then vertical position is determined by integrating the vertical speed obtained by Eq. (11).

$$
\dot{z}_{r i}=\dot{z}_{w i}+b b_{i} \dot{\theta}_{w i}-\left(\left(\dot{y}_{w i}+r_{i} \dot{\theta}_{w i}\right)-\dot{y}_{r i}\right) \tan \left(\alpha_{i}\right)
$$

\section{Conditions for simulation}

\subsection{Specifications and inputs}

The specification parameters for the numerical calculations are given basically based on the typical parameters of a Japanese high speed train as shown in Table 1.

Table 1 Specification parameters for 13 DoF model

\begin{tabular}{|cc|cc|}
\hline & {$[\mathrm{kg}]$} & & {$[\mathrm{kN} / \mathrm{m}]$} \\
\hline$M_{b}$ & $1.3 \mathrm{E}+04$ & $k_{1}:\left|z_{1 i}\right|<25 \mathrm{~mm}$ & $1.2 \mathrm{E}+3 / \mathrm{box}$ \\
$M_{t}$ & $3.2 \mathrm{E}+03$ & $k_{1}:\left|z_{1 i}\right| \geqq 25 \mathrm{~mm}$ & $3.0 \mathrm{E}+4 / \mathrm{box}$ \\
$M_{w}$ & $1.8 \mathrm{E}+03$ & $k_{2}: z_{2 i} \geqq-35 \mathrm{~mm}$ & $2.7 \mathrm{E}+02$ \\
\cline { 1 - 2 } & {$[\mathrm{m}]$} & $k_{2}: z_{2 i}<-35 \mathrm{~mm}$ & $6.0 \mathrm{E}+03$ \\
\cline { 1 - 2 }$I_{b x}$ & 1.20 & $k_{3}:\left|y_{3}\right|<40 \mathrm{~mm}$ & $1.1 \mathrm{E}+02$ \\
$I_{t x}$ & 0.70 & $k_{3}:\left|y_{3}\right| \geqq 40 \mathrm{~mm}$ & $6.0 \mathrm{E}+03$ \\
$I_{w x}$ & 0.75 & & \\
\hline
\end{tabular}

The input excitation is imposed to the ground of the vehicle-track system in the roll plane as a horizontal sine displacement, which is orthogonal to the vehicle forwarding direction. As shown in Fig.6, the input is a three-cycle sine wave and has relaxations at the start and end to reduce shocks in the dynamic system due to the discontinuities. Note that significant 
events such as large wheel lift and derailment occur within the first three cycles, thus we could say three-cycle steady state is sufficient to analyze the vehicle motions. Various frequencies and amplitudes of sine inputs are selected to understand the fundamental characteristics of vehicle motions as the dynamics issue of forced vibrations. By considering the natural frequencies of the coupled motions of vehicle body lateral and roll are given at 0.54 and $0.97 \mathrm{~Hz}$, the frequency range of 0.5 to $2.0 \mathrm{~Hz}$ is applied for excitation frequencies.

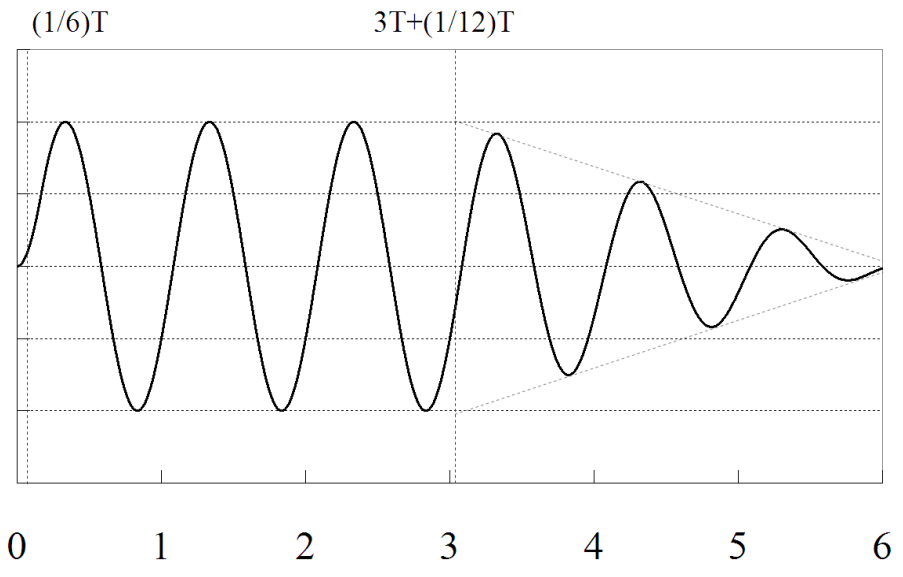

Fig.6 Shape of an excitation input

\subsection{Definition of derailment}

The motions of the excited vehicle could significantly differ from those of a vehicle under a normal operation. The vehicle body might sway severely and the wheels could lift off considerably. In general, the safety of rail vehicles is assessed by the derailment coefficient or the wheel load reduction ratio; however, in this study, we should apply a more direct definition of derailment. Thus, to judge derailment in our numerical results, we employ the definition that the wheel vertically lifts to flange height $(30 \mathrm{~mm})$ and laterally slides $70 \mathrm{~mm}$ from the initial regular position as shown in Fig.7, which is proposed in Ref[11].

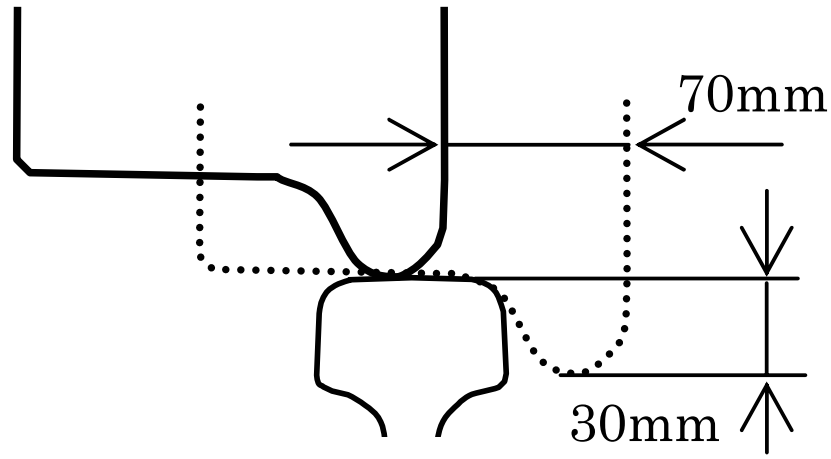

Fig.7 Diagrams of derailment

\section{Numerical results and analysis}

Based on the results of the numerical calculation, both the derailment mechanism such as derailment patterns, behaviors and amplitudes, and the characteristics of wheel lift including amplitudes for $30 \mathrm{~mm}$ wheel lift are studied. Also, the effect of vehicle speed is analyzed through the numerical results. 


\subsection{Effect of vehicle speed on the derailment mechanism}

We examine the effect of vehicle speed on the derailment mechanism by changing the input excitation of amplitude and frequency at different vehicle speeds $(0 \mathrm{~km} / \mathrm{h}$ and $300 \mathrm{~km} / \mathrm{h}$ ) as shown in Fig.8 and Fig.9. The results are calculated under the conditions of either the input displacement of less than $\pm 0.4 \mathrm{~m}$ or the input acceleration of less than \pm 1500 gal.

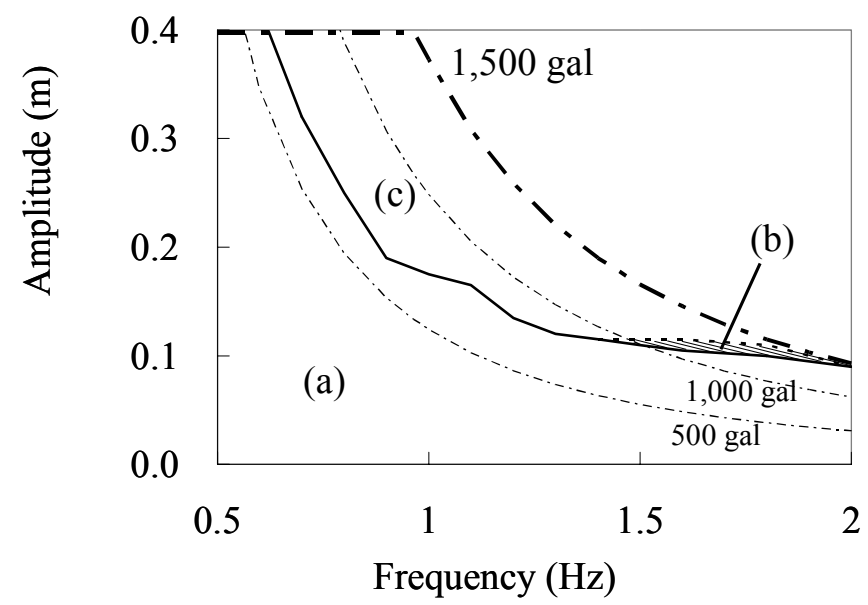

Fig.8 Excitation amplitudes for derailment $(\mathrm{V}=0 \mathrm{~km} / \mathrm{h})$

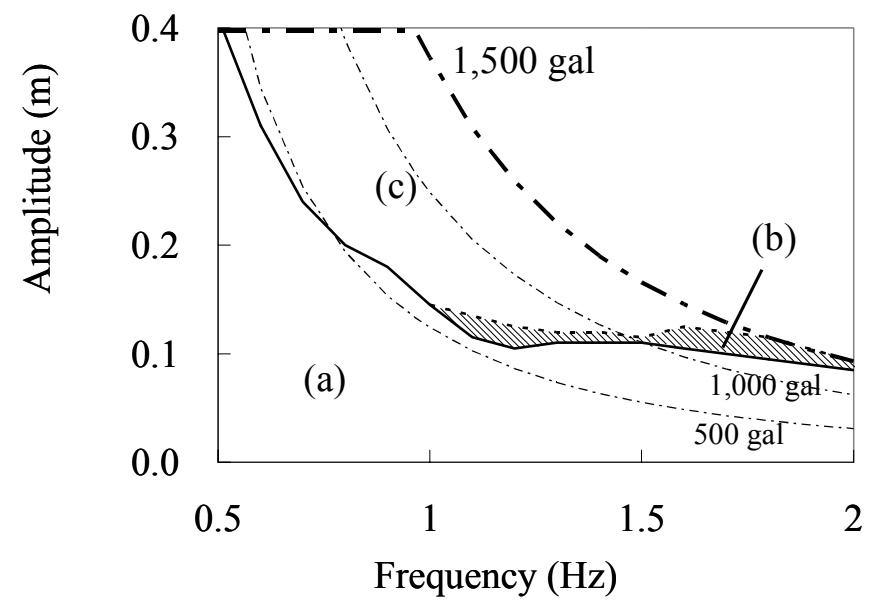

Fig.9 Excitation amplitudes for derailment ( $V=300 \mathrm{~km} / \mathrm{h})$

In both of Fig.8 and Fig.9, by the excitations in the area of (a) below the solid line, the vehicles are safe, thus not derailed. On the other hand, by the excitations that exceed the solid line, the vehicles are derailed. As with derailment patterns, two types are observed; one is "sliding derailment" in the area of (b) and the other is "rocking derailment" in the area of (c). During the process of sliding derailment, a wheel makes impacts upon a rail, slides up on the rail shoulder, and then moves toward outside of the track and finally satisfies the defined condition of derailment. By contrast, during the process of rocking derailment, in some cases, a wheel once lifts up, then drops down on the rail at flange top and slides toward the outside of the track. In other cases, a wheel lifts up higher and during the lifting, it crosses over the rail and then drops down directly outside of the track. We judge the possibility of rocking derailment to be far more likely than that of sliding derailment by comparing the area for both derailment types shown in Fig.8 or Fig.9. Based 
on the analysis, it could be basically mentioned that most of derailment is caused by the rocking derailment regardless of vehicle speeds, even though the possibility of sliding derailment seems to increase slightly as vehicle speeds get higher.

Next, we demonstrate the two derailment processes (rocking derailment and sliding derailment) based on the case of the vehicle speed being $100 \mathrm{~km} / \mathrm{h}$. The excitation changes and wheel vertical and lateral motion time history of wave forms are as shown in Fig.10 and Fig.11. Firstly, as shown in Fig.10, for the case of rocking derailment, wheels No.2 and No.1 lift up alternatively. Just after $2.2 \mathrm{~s}$, while wheel No.2 is lifting, the wheel starts to slide toward the outside of the track, and then drops down on the rail at the flange top at $2.4 \mathrm{~s}$ continuing lateral wheel/rail slide, and finally satisfies the derailment condition. Secondly, as shown in Fig.11, for the case of sliding derailment, at $1.2 \mathrm{~s}$ wheel No.1 starts to lift, at $1.3 \mathrm{~s}$ the wheel horizontally makes impact on the rail and slides up on the rail shoulder and just beyond 1.4s finally satisfies the derailment condition. Note that at the moment of derailment in both cases, the wheel opposite to the derailing one is in contact with the rail.

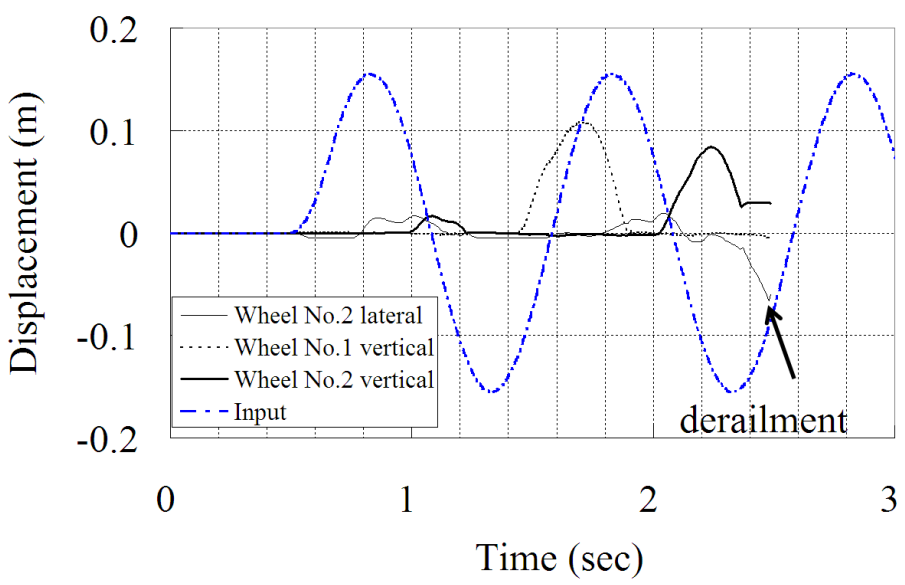

Fig.10 Time histories of rocking derailment (Input: $1.0 \mathrm{~Hz} \pm 160 \mathrm{~mm}$ )

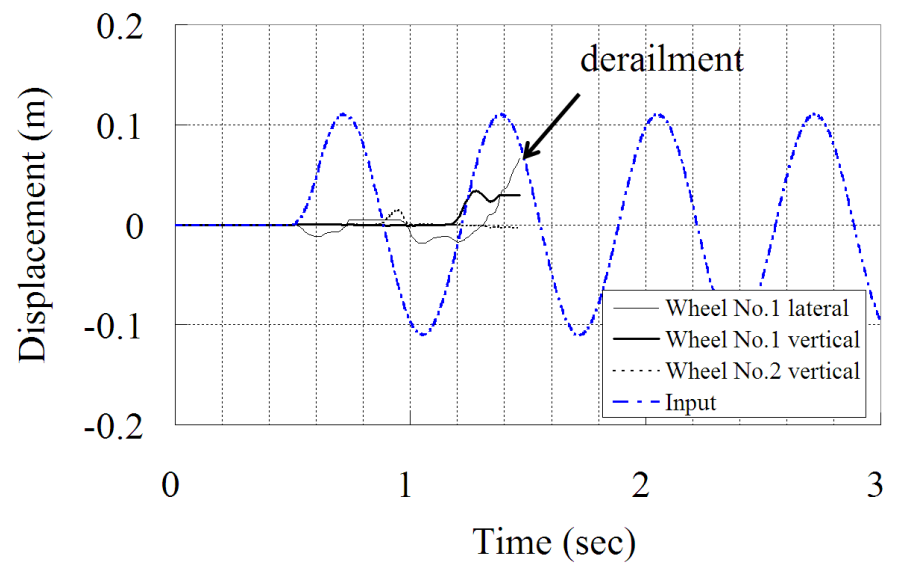

Fig.11 Time histories of sliding derailment (Input: $1.5 \mathrm{~Hz} \pm 110 \mathrm{~mm}$ )

\subsection{Effect of vehicle speed on derailment limit}

Figure 12 demonstrates the effect of vehicle speed on derailment. Regarding the fundamental effect, the derailment amplitudes decrease as vehicle speed increases, and then the weakening trend gradually decreases, and finally stays almost steady at higher vehicle speeds. This could be anticipated as indicated in Fig. 8 and Fig. 9: a vehicle is not derailed 
at $0 \mathrm{~km} / \mathrm{h}$ if the excitation acceleration is not greater than $500 \mathrm{gal}$; however, a vehicle could be derailed at $300 \mathrm{~km} / \mathrm{h}$ even if the excitation acceleration is smaller than 500 gal. Figure 12 also demonstrates that the decreasing trend of the derailment limit is relatively clear in the input frequency range of less than $0.8 \mathrm{~Hz}$, and not explicit in the high frequency range, for example beyond $1.5 \mathrm{~Hz}$.

The basic mechanism for the decrease of the derailment limit is attributed to the speed dependency of wheel/rail lateral slide; that is, a rotating wheel has a basic tendency to slide more with an increase of vehicle speed by even equal lateral force given based on the creep law. Because of the excitations at lower frequency, the forces acting between the wheel/rail becomes smaller and far from the saturation with a fixed creepage, thus the effect becomes clearer in lower frequency cases. To explain it by numerical result, the time history of vehicle motions by excitation of $0.8 \mathrm{~Hz} \pm 200 \mathrm{~mm}$ at $0 \mathrm{~km} / \mathrm{h}$ and $300 \mathrm{~km} / \mathrm{h}$ cases are shown in Fig.13, such as wheel vertical and lateral positions.

Just beyond $2.5 \mathrm{~s}$, while wheel No.2 is lifting, wheel No.2 slide at $300 \mathrm{~km} / \mathrm{h}$ is much greater than that at $0 \mathrm{~km} / \mathrm{h}$. Thus, in case of $300 \mathrm{~km} / \mathrm{h}$, the wheel-set slides significantly and reaches the definition of derailment.

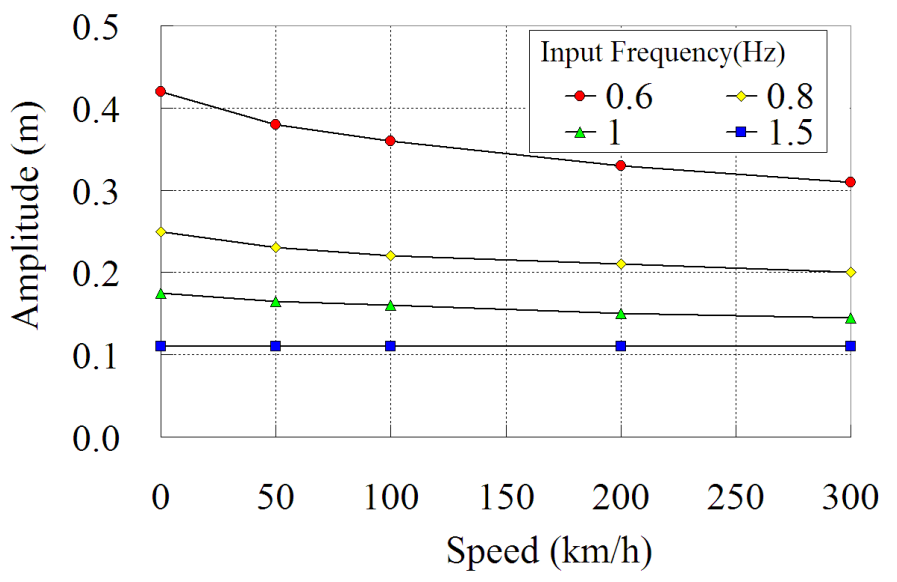

Fig.12 Effect of vehicle speed on derailment limit

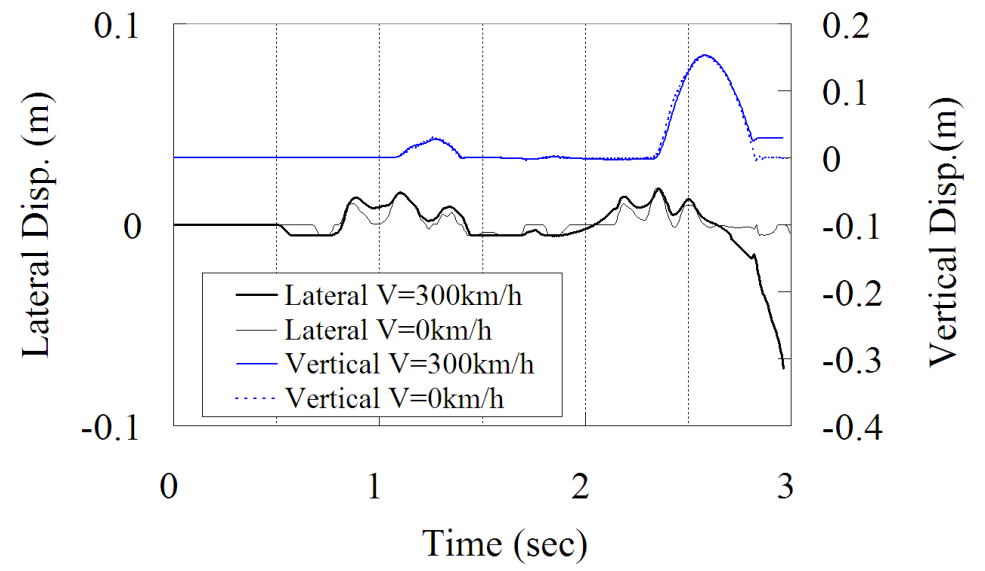

Fig.13 Comparison of time histories of wheel No.2 motions

\subsection{Effect of vehicle speed on wheel lift}

Figure 14 demonstrates the limit amplitudes for $30 \mathrm{~mm}$ wheel lift at the speed of $0 \mathrm{~km} / \mathrm{h}$ and $300 \mathrm{~km} / \mathrm{h}$. Although as shown in Fig.8 and Fig. 9, the derailment amplitudes monotonously decrease as input frequency increases, the $30 \mathrm{~mm}$ wheel lift amplitudes shows unique trends in relation to the input frequency; that is, it basically has a general decreasing trend as frequency increases and has the local minimum around $1.0 \mathrm{~Hz}$. The trend of 
frequency dependency could be attributed to the coupling of vehicle motion of vehicle body roll and wheel lift. The similar trend and mechanism were pointed out by in the analytical work based on the simple 3 DoF model suggested in Ref[9]. Figure 15 shows the vehicle body roll ratio to the input displacement of $100 \mathrm{~mm}$ over the frequency range of $0.5 \mathrm{~Hz}$ to $1.6 \mathrm{~Hz}$. The frequencies of the local minimum amplitude of $30 \mathrm{~mm}$ wheel lift and the local maximum response of body roll ratio are seen to be very close, which indicates that both phenomenon have significant correlation.

Figure 14 demonstrates that the effect of vehicle speed on wheel lift motion should be small. Note that the effect of vehicle body roll on wheel lift motion should be significant; by contrast, the effect of wheel/rail slide on wheel lift motion discussed in 5.2 should be small.

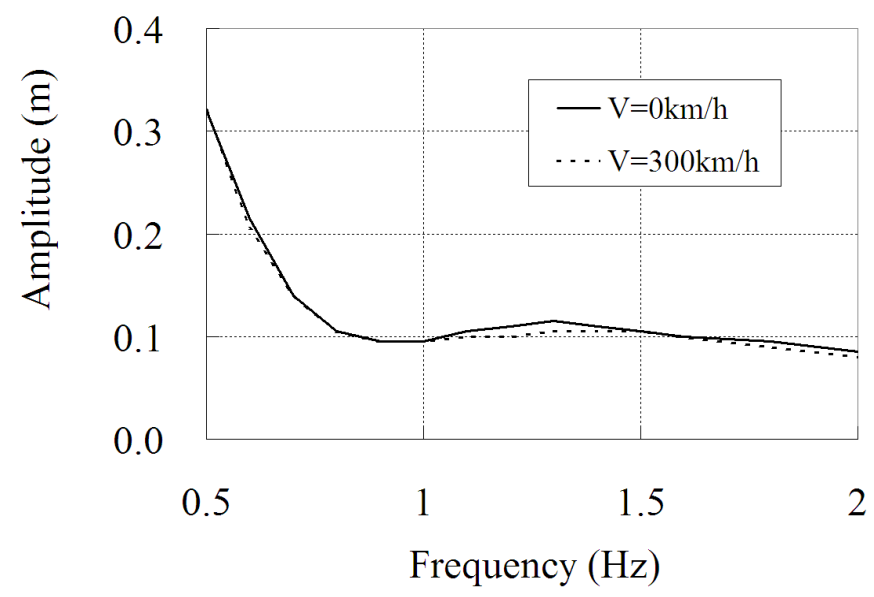

Fig.14 Excitation amplitudes for 30mm wheel lift

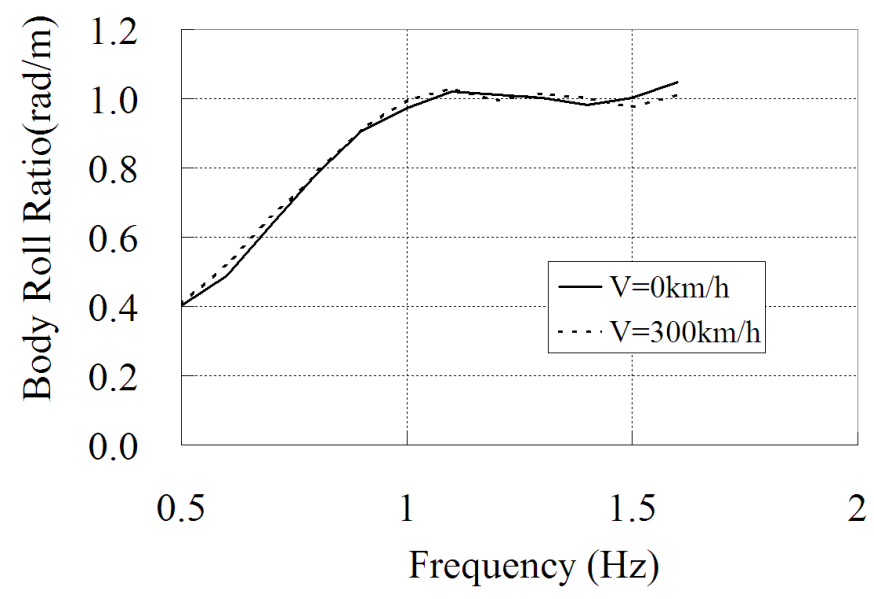

Fig.15 Body roll response to input amplitude

\subsection{Effect of friction coefficient on wheel/rail slide}

We found that the rocking derailment is the main pattern of derailment in those cases and the derailment is basically caused by both phenomenon; (1) wheel lift strongly related to vehicle body roll by the excitation of ground displacement and (2) wheel lateral slide because of the acceleration of excitation. Based on the analysis, the effect of vehicle speed is small on (1) wheel lift, but significant on (2) wheel slide, which yields the speed dependency of derailment.

As a high speed rail vehicle is normally operated at very high speeds, it is important to examine a potential factor which could influence wheel/rail slide. To examine the influence of the friction coefficient on wheel/rail slide, friction coefficients of different magnitude are 
set and analyzed. Figure16 shows the time history of wheel/rail lateral relative displacement by the excitation of $1 \mathrm{~Hz} \pm 70 \mathrm{~mm}$, at the speed of $0 \mathrm{~km} / \mathrm{h}$, with three cases of the friction coefficient $0.3,0.2$ and 0.1 . In case of 0.3 , the displacement is quite small because the wheel adheres to the rail; on the other hand, in cases of 0.2 and 0.1 , the displacements become larger implying more slips occur in the wheel/rail contact patch. Thus, in case of the speed of $0 \mathrm{~km} / \mathrm{h}$, the friction coefficient has a significant influence on wheel $/$ rail slide and a smaller coefficient leads to larger slide.

Next, Fig. 17 shows the time history of wheel/rail lateral relative displacement by the excitation of $1 \mathrm{~Hz} \pm 70 \mathrm{~mm}$, at the speed of $300 \mathrm{~km} / \mathrm{h}$, with three cases of the friction coefficient $0.3,0.2$ and 0.1 . In those cases, regardless of the magnitude of the coefficient, the displacements are quite close. Thus, in case of very high speed, the friction coefficient does not seem to play a significant roll in wheel/rail slide.

By comparing the cases of still and very high speed, for a friction coefficient of 0.1 , the wheel lateral motions are very similar; by contrast, for a friction coefficient of 0.2 and 0.3 , the wheel lateral motions are smaller and the wheel starts to slide more slowly. Therefore, in terms of wheel/rail lateral slide, if the vehicle speed is very high, the influence of friction coefficient is smaller; however, if the vehicle speed is small, the influence of the friction coefficient becomes larger. Note that even if the vehicle speed is small, if the friction coefficient becomes small, i.e. 0.1 , then the situation of wheel/rail slide could be similar to that of higher speed case.

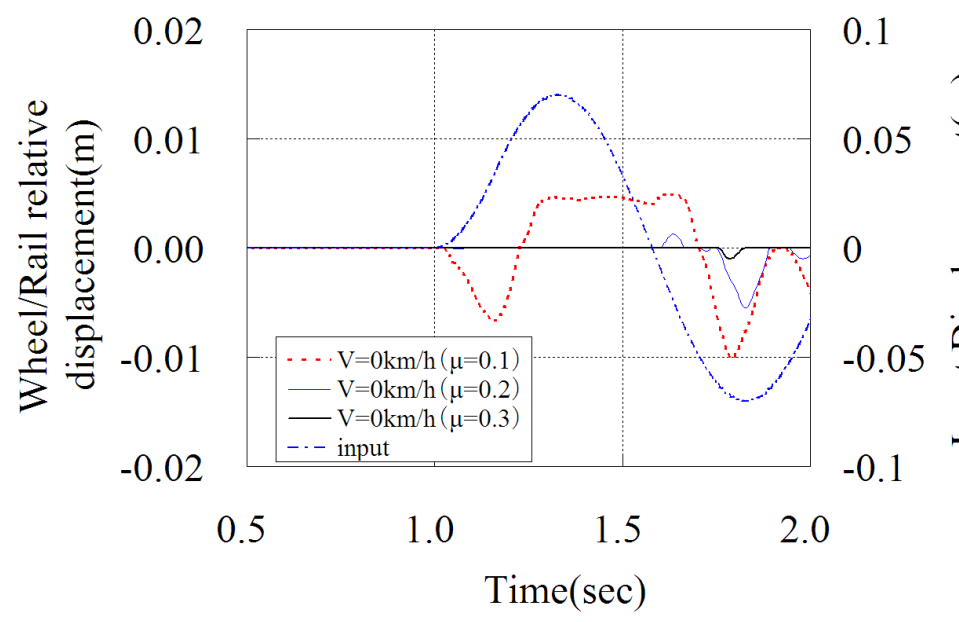

Fig.16 Effect of friction coefficient at $\mathrm{V}=0 \mathrm{~km} / \mathrm{h}$

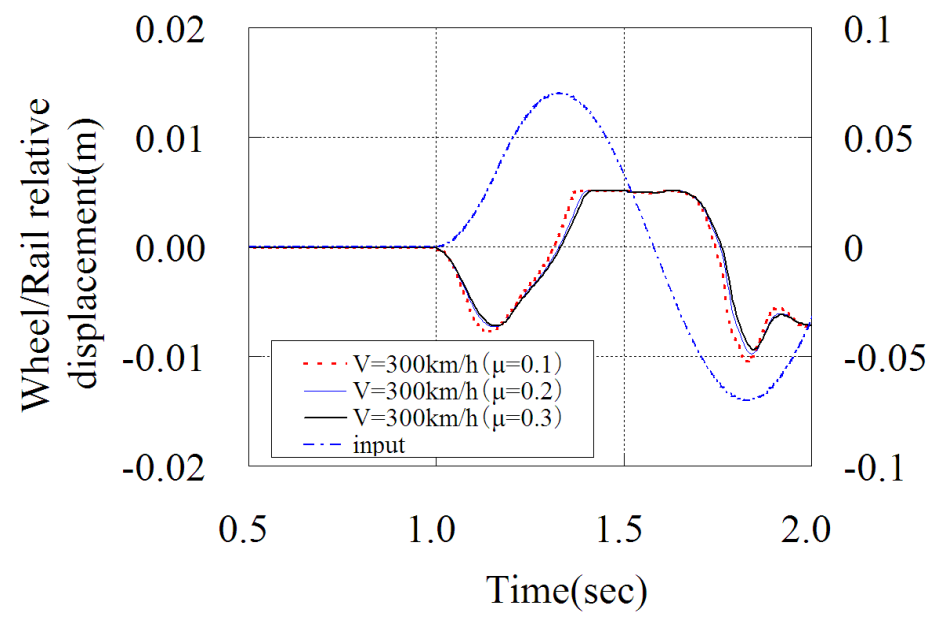

Fig. 17 Effect of friction coefficient at V=300 km/h 


\section{Conclusions}

Based on the facts and reports which indicate that rail vehicles could be derailed solely by the ground motion of earthquakes with no fatal damages to vehicles and tracks, we believe that we should further study the derailment mechanism of a high speed railway vehicle excited by large seismic motions both theoretically and experimentally. Therefore, in this study, we developed our simulation program and analyzed the derailment mechanism and effect of vehicle speed on it based on the numerical results with sine wave excitations. The following outcomes were obtained.

(1) Rocking derailment is the main derailment pattern due to large track excitations. The derailment is basically caused by both phenomenon; (a) wheel lift strongly related to vehicle body roll by the excitation of ground displacement and (b) wheel lateral slide by the acceleration of excitation. Vehicle speed has no significant influence on derailment patterns.

(2) The derailment amplitudes decrease as vehicle speed increases, and then the decreasing trend gradually weakens and finally stays almost steady at higher vehicle speeds. The basic mechanism for the decrease of the derailment limit is attributed to the speed dependency of wheel/rail lateral slide. Given a constant lateral force between wheel and rail, a wheel has a basic tendency to slide more and faster with the increase of vehicle speed based on the creep law.

(3) The effect of vehicle speed is small on wheel lift appearing in the process of vehicle rocking motion.

(4) The effect of the wheel/rail friction coefficient on wheel/rail slide is small in the case of very high speed but significant for the case of a still vehicle.

Even assuming no fatal damages to tracks and two dimensional vehicle motions in roll plane, we could find the fundamental derailment mechanism and safety limit of a high speed vehicle based on our original numerical simulation.

For our next step in the future, we shall verify the simulation model by comparing test results and expand the model for more precise analysis, and discuss the cases of vehicle responses by the excitations of measured earthquake ground motion. Through such work, we shall pursue complete and comprehensive understanding of the derailment mechanism of high speed rail vehicles due to large earthquakes.

\section{References}

(1) Nagase, K., Kondo, K. and Nomura, T., 1997, "Train Damaged by the Quake in kobe", Transactions of the Japan Society of Mechanical Engineers, Series C, Vol. C-63-606, pp 300-307

(2) Aircraft and Railway Accidents Investigation Commission, 2007, "The Report of the Investigation on the Derailment Accident at Jyouetsu Shinkansen of JR East", pp1-56

(3) Ishida, H. and Matsuo, M., 1999, "Safety Criteria for Evaluation of Railway Vehicle Derailment”, RTRI Quarterly Report, Vol. 40 No.1

(4) Miyamoto, M., 1996, "Mechanism of Derailment Phenomena of Railway Vehicles", RTRI Quarterly Report, Vol. 37 No.3 pp147-155

(5) Matsudaira, T. and Yokose, K., 1962, "Derailment Caused by the Impacting of Wheel to Rail", Research for Tokaido Shinkansen, Vol.3 pp 235-244

(6) Yokose, K., 1970, "Experiment of Hunting Derailment with a One-fifth Model Wheel-set”, RTRI Quarterly Report, Vol.11-4 pp228-231

(7) Matsuo, M. and Muroi, Y., 1990, "Derailment under Oscillatory Wheel load Fluctuation”, RTRI report, Vol.4-6, pp 8-15

(8) Ishida, H. and Matsuo, M., 2004, "Analysis on Dynamic Behavior of Railway Vehicle 
Wheel-set Running on Wavy Rails at High Speed", RTRI report, Vol.18-8, pp 11-16

(9) Nihon Tetsudou Shisetsu Kyoukai, 1982, "Safety Assessment for Derailment of Rail Vehicle on Excited Tracks", Research on rail vehicle safety for operation on the connecting bridge between Japan Mainland and Shikoku Island, Soukoubunkakai Houkokushyo pp194-204

(10) Kunieda, M., Karasawa, A., Udagawa, K., and Nishimura, S., 1981, "Theoretical Study on Overturn Motions of Rail Vehicles on Earthquakes", Transactions of the Japan Society of Mechanical Engineers, Series C, Vol. C-47-414, pp 164-170

(11) Miyamoto, T., Ishida, H., and Matsuo, M., 1998, "The Dynamic Behaviors of Railway Vehicles during Earthquake", Transactions of the Japan Society of Mechanical Engineers, Series C, Vol. C-64-626, pp 236-243

(12) Nishimura, K., Terumichi, Y., Morimura, T. and Sogabe, K., 2007, "Development of Vehicle Dynamics Simulation for Safety Analyses of Rail Vehicle on Excited Tracks", Proceedings of the ASME 2007 IDETC, DETC2007-34474 